\title{
Third harmonic current minimization using third harmonic blocking transformer
}

\author{
Vishnuprasada Vittal Bhat, Pinto Pius
}

Department of Electronics and Electrical Engineering, NMAMIT, Nitte, India

\begin{tabular}{|c|c|}
\hline Article Info & ABSTRACT \\
\hline Article history: & Zero sequence blocking transformers (ZSBTs) are used to suppress third \\
\hline Received Sep 1, 2021 & $\begin{array}{l}\text { harmonic currents in } 3 \text {-phase systems. Three-phase systems where single- } \\
\text { phase loading is present, there is every chance that the load is not balanced. }\end{array}$ \\
\hline Revised Dec 22, 2021 & If there is zero-sequence current due to unequal load current, then the ZSBT \\
\hline Accepted Dec 29, 2021 & $\begin{array}{l}\text { will impose high impedance and the supply voltage at the load end will be } \\
\text { varied which is not desired. This paper presents Third harmonic blocking }\end{array}$ \\
\hline Keywords: & $\begin{array}{l}\text { transformer (THBT) which suppresses only higher harmonic zero sequences. } \\
\text { The constructional features using all windings in single-core and }\end{array}$ \\
\hline Industrial power systems & construction using three single-phase transformers explained. The paper \\
\hline Power distribution lines & $\begin{array}{l}\text { discusses the constructional teatures, full detalls of circuit usage, design } \\
\text { considerations, and simulation results for different supply and load }\end{array}$ \\
\hline $\begin{array}{l}\text { Power harmonic filters } \\
\text { Power quality }\end{array}$ & $\begin{array}{l}\text { conditions. A comparison of THBT with ZSBT is made with simulation } \\
\text { results by considering four different cases. }\end{array}$ \\
\hline
\end{tabular}

This is an open access article under the CC BY-SA license.

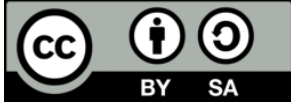

\section{Corresponding Author:}

Vishnuprasada Vittal Bhat

Department of Electronics and Electrical Engineering, NMAMIT

Nitte, India

Email: vishnupangala@gmail.com

\section{INTRODUCTION}

Power quality is very important in a power system. The removal of the third harmonic is very difficult as it is near to fundamental. There are different technologies to remove third harmonics. The below discussion reveals problems with different technologies:

a) Using an inductor in series with the line can be implemented for individual loads. If implemented for industrial loads where both single-phase and three-phase loads are present, due to unequal single-phase loads or unbalanced loads, the voltage drop in phase will be unequal and hence supply voltage also varies that causing further unbalance which is not desirable. As the entire phase current flows through the inductance, the copper loss is also more.

b) If ZSBT [1]-[4] is used, power loss is less. But if an unbalanced current (zero-sequence current) flows due to single-phase loading, the voltage drop in lines will be different, and hence even if the three-phase balanced load is present, it causes increased unbalanced current.

c) A star-delta transformer [5] or a zigzag transformer [6] can be connected in parallel with a load but if a very small zero sequence component is present in supply voltage, then it will draw a very high current which causes more losses.

d) Active filters/active line conditioners [7] are the better option but are more complex and the cost is also higher compared to passive filters. Also, they are not reliable as compared to passive filters.

Differential protection is the most effective protection against internal defects for power transformers. The protection uses differential currents, which differ from the primary currents to the second one [8], [9]. The differential relay is utilized to protect the transformer, alternator, and HT motor. If the 
transformer winding faults, the relay will trip. The differential relay must remain inoperative when the problem is outside of the area. The differential relay should detect energy inrush and prohibit operation. The relay must also be temporarily removed from operation to allow the transformer to be placed in service. This is not an option in the majority of cases. The harmonics are usually small in faults. The second harmonic, on the other hand, is a key part of the inrush current. The second harmonic thus offers an efficient technique of differentiating between faults and inrush [10], [11]. Inrush currents are produced by transients in the magnetic transformers before the flux is stable [12]. The following include early efforts to prevent differential relay operations resulting from inrush:

- Introduce a delay in the differential relay intentionally [13].

- The relay has been desensitized for a period to override inrush.

- To restrict or govern the differential relay, add a voltage signal.

When a transformer is energized, transient current may appear up to 10 to 15 times more than the rated transformer current. These inrush currents can continue for a few seconds for big transformers until the transient is lost. Transformer inrush is a high current, affecting the surrounded device's lifetime on the network and creating protection selection and stability difficulties. This phenomenon must therefore be included in the calculation of relay settings [14]. Hence, we need a system that should be very simple, it should have fewer/no active components and ideally, it should have high impedance for only harmonic current and no effect on fundamental currents.

Jiang et al. [15] have designed a harmonic blocking system to send the GOOSE-based harmonic blocking signal to the backup current relay (SEL751A) via the element (87HB) of the transformer differential relay to prevent from tripping during the transformer magnetizing inrush current conditions. The results of the simulation demonstrate that the default protection system IEC61850 is faster than the hardwired signals. The transformer system hence improves speed and reliability using the standard GOOSE application IEC61850.

Shaikh et al. [16] proposed a zigzag transformer to minimize the harmonic currents and the neutral overload. The various nature of the loads connected to the four-phase three-wire distribution system. These may include personal computers, automatic machines, variable speed drives, lighting ballasts, and other electronic power systems, which can cause zero sequence current in the system to fluctuate in the neutral supply system. This could lead to major problems of power quality and reduction. One method for reducing the neutral current and for reducing the harmonic zero sequence currents of distributive systems is a zigzag transformer. Rahman et al. [17] examined the variation of THD for the THPWM technology by modifying the carriers' frequency and index of modulation. The research suggests an optimal condition that provides the lowest THD for that ideal state. A modified THPWM approach is suggested in this research. This proposed method of modulation helps to minimize the THD to the lowest value than the approach THPWM.

Eltamaly [18] introduced a new current injection system for harmonically reducing renewable energy utility interface three-phase controlled converters. In renewable energy applications such as photovoltaics and wind energy, three-phase controlled or uncontrolled converters have been applied as rectifiers and inverters. The fundamental disadvantage of these converters is that their line currents are high THD. This difficulty was remedied by many approaches. One of the greatest options for a harmonic reduction of these converters' line currents is a third harmonic current injection. The injection into the line currents of the third harmonic current from dc-bus decreases its harmonic contents.

Almutairi and Hadjiloucas [19] has been proposed a new method for the suppression of distortions, for non-sinusoidal power systems to use the non-linearity current index (NLCI) to define the shunt singletune passive filter (STPF). The aim was to maintain a power factor within the required range. The purpose of the approach is to minimize the nonlinear current of customer's loads at the point of common coupling in the power system (PCC). Gbadamosi et al. [20] focuses on finding an optimum model of power flow optimization to minimize power loss and RES harmonics with a concentration on minimizing their effects on local marginal prices. The electrical transient analyzer program 12.6.0 has been used to evaluate the magnitude of harmonics, grid modeling, and simulation using RES, such as wind and solar energy.

Arafat and Choi [21] introduced a five-stage permanent magnet assisted synchronous reticence motor (PMa-SynRM) for the smart and adaptive performance of torque ripple minimization (TRM) in openstage faults. Due to its potential fault tolerance and wide-speed operation capabilities, the five-phase machine is gaining more attention. Furthermore, the torque ripple is significantly enhanced by harmful vibrations during fault tolerance. Yet, the majority of TRM has been undertaken simply to preserve continuous MMF while evaluating its fundamental harmonic. But even huge torque variations are induced by suddenly modulated harmonic oscillations of a high order which are uncertain.

Routray et al. [22] proposed a modified particle swarm optimization (MPSO) in the three-phase eleven-level hybrid cascaded multilevel inverter (HC-MLI) for rapid convergence and harmonic minimization. In the proposed work on the synthesis of an eleven-level output voltage employing two dc 
sources, a pre-charged capacitor, and twelve switches, Selective harmonic elimination pulse width modulation (SHE-PWM) was performed. To remove the lower-order odd harmonics like 5th, 7th, 11th, and 13th from the HCMLI output voltage, the three-phase eleven-level HC-MLI switching angles were determined.

Yadav et al. [23] studied the transformerless function of the MMC. The MMC's internal dynamic produces a third-order voltage ripple, which could create a harmonic current of the third order. This harmonic current is prevented with star-delta transformers as an interface between MMC and AC network. This third level of harmonic current, however, causes serious damage to the MMC and the AC source with the transformerless MMC and physically grounded DC terminal. This work also suggests the suppression of the third-order harmonic current by a proportional resonant $(\mathrm{PR})$ controller.

This paper proposes a new technology to remove third harmonic currents. The major contributions of the proposed paper are:

- A passive filter that blocks zero-sequence harmonics [24] especially the third harmonic is presented. The construction, working principle, and design considerations are discussed.

- The simulation is carried for four cases each with THBT, with ZSBT, and without a filter. The results are discussed. The applicability of THBT over ZSBT in the distribution network or industrial loads is presented.

- The discussed filter imposes very low impedance [25] for fundamental components even if zero sequence components are prlesent. The filter can be used in industrial loads or distribution networks where along with three-phase load single-phase load is also present, due to which zero sequence current is more frequent.

- The filter suppresses the surge current due to non-linear loads. The cost of the filter is less compared to another kind of filter. Also, the filter is more reliable as it contains only passive elements.

\section{METHOD}

\subsection{Working principle}

THBT comprises four mutual inductances as shown in Figure 1. Three inductances are connected in series with every three phases of supply and the fourth winding is connected with a filter. Figure 2 shows the circuit connection of THBT with single-phase and three-phase loads. THBT can be considered as a transformer with three primary windings and one secondary winding. Let the secondary be open-circuit and the primary three windings are in series with three phases for the analysis.
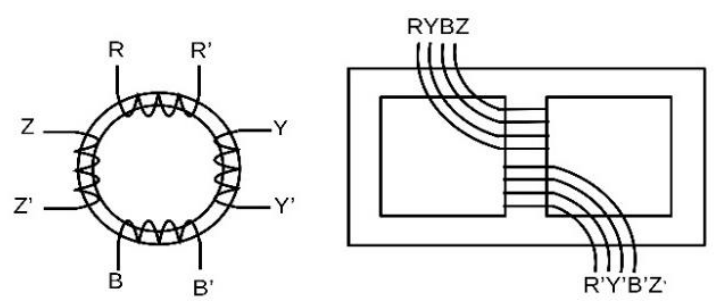

Figure 1. THBT winding

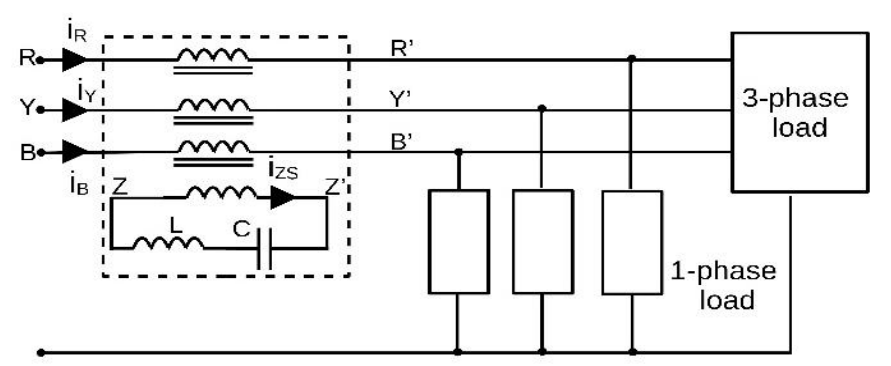

Figure 2. THBT circuit diagram

Consider $i_{r}, i_{y}$ and $i_{b}$ be the phase currents and $i_{z s}$ be the zero-sequence current. Let $v_{r}, v_{y}$, and $v_{b}$ be the voltage across three windings of the THBT, $v_{0}$ be the voltage across the fourth winding. Let $R_{w r}$, $R_{w y}$, and 
$\mathrm{R}_{\mathrm{wb}}$ be the winding resistance of coils connected in series with phases $\mathrm{R}, \mathrm{Y}$, and $\mathrm{B}$ respectively. Let $\mathrm{L}$ be the self-inductance of three windings and $\mathrm{L}_{0}$ be the mutual inductance.

$$
v_{r}=L \frac{d i_{r}}{d t}+L_{0} \frac{d i_{y}}{d t}+L_{0} \frac{d i_{b}}{d t}+R_{w r} * i_{r}
$$

In (1) can be written as (2),

$$
v_{r}=L_{l k} \frac{d i_{r}}{d t}+L_{0} \frac{d i_{r}}{d t}+L_{0} \frac{d i_{y}}{d t}+L_{0} \frac{d i_{b}}{d t}+R_{w r} * i_{r}
$$

where $\mathrm{L}_{\mathrm{lk}}=\left(\mathrm{L}-\mathrm{L}_{0}\right)$ is leakage inductance.

$$
v_{r}=L_{l k} \frac{d i_{r}}{d t}+L_{0} \frac{d\left(i_{r}+i_{y}+i_{b}\right)}{d t}+R_{w r} * i_{r}
$$

By definition of zero sequences, $i_{z s}=\frac{\left(i_{r}+i_{y}+i_{b}\right)}{3}$ (3) can be re-written as (4).

$$
v_{r}=L_{l k} \frac{d i_{r}}{d t}+3 L_{0} \frac{d\left(i_{z s}\right)}{d t}+R_{w r} * i_{r}
$$

Similarly, for other phases, it can be derived as (5) and (6).

$$
\begin{aligned}
& v_{y}=L_{l k} \frac{d i_{y}}{d t}+3 L_{0} \frac{d\left(i_{z s}\right)}{d t}+R_{w y} * i_{y} \\
& v_{b}=L_{l k} \frac{d i_{b}}{d t}+3 L_{0} \frac{d\left(i_{z s}\right)}{d t}+R_{w b} * i_{b}
\end{aligned}
$$

Since mutual inductance is much higher than leakage inductance and if properly designed the winding resistance will be negligible. Hence (4), (5), and (6) can be re-written as (7).

$$
v_{r}=v_{y}=v_{b}=3 L_{0} \frac{d\left(i_{z s}\right)}{d t}
$$

Since the symmetric components (positive and negative sequences) are balanced sets of sinusoids with 120 phase-shifted each other, the sum of instantaneous currents is zero and hence the flux generated is also zero. Hence the symmetric components (positive and negative sequences) have very low impedance

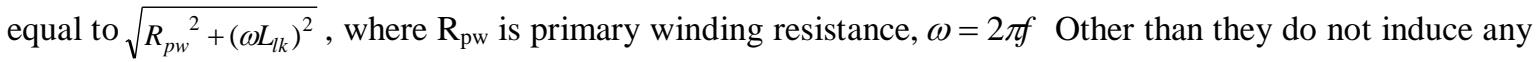
voltage in the primary or secondary side of the inductance. Similar to (4) to (6) the output voltage can be derived as (8).

$$
v_{0}=3 K L_{0} \frac{d\left(i_{z s}\right)}{d t}
$$

Where $K=\frac{N_{2}}{N_{1}}$ turn ratio. The secondary side can be any low pass filter for currents that means it should have low impedance for fundamental current and high impedance for high-frequency currents. In this paper, the low pass filter is taken as an LC series circuit. Consider inductor $\mathrm{L}$ is in series with capacitor $\mathrm{C}$ which is connected across the output/secondary side of the transformer as shown in Figure 2. The value of $\mathrm{L}$ and $\mathrm{C}$ are selected such that $f=\frac{1}{2 \pi \sqrt{L C}}$ where $\mathrm{f}$ is the fundamental supply frequency which is equal to resonating frequency of $\mathrm{L}$ and $\mathrm{C}$. The impedance is ideally zero at resonating frequency but practically it will be equal to the sum of winding resistance of a secondary winding of THBT $R_{w r}$, winding resistance of $R_{1}$ of inductance $\mathrm{L}$, and series resistance $\mathrm{R}_{\mathrm{c}}$ of capacitor $\mathrm{C}$. However, these resistances are negligibly small.

$$
\begin{aligned}
& R_{s}=R_{w r}+R_{l}+R_{c} \\
& Z_{s f l}=2 \pi f L \\
& Z_{s f c}=\frac{1}{2 \pi f C}
\end{aligned}
$$


At resonating frequency;

$$
Z_{s f}=\sqrt{R^{2}+\left(Z_{s f l}-Z_{s f c}\right)^{2}}=R_{S}
$$

where $Z_{s f l}$ is the magnitude of the impedance of inductance $L$ for fundamental components, $Z_{\text {sfc }}$ is the magnitude of the impedance of capacitor $C$ for fundamental components, $Z_{s}$ is the total magnitude of impedance for fundamental components. For the third harmonic which is the dominant component in zerosequence other than fundamental, the magnitude of impedance can be calculated as:

$$
\begin{aligned}
& Z_{s 3 h l}=3 Z_{s f l} \\
& Z_{s 3 h c}=\frac{Z_{s f c}}{3}=\frac{Z_{s f l}}{3} \\
& Z_{s 3 h}=\sqrt{{R_{s}}^{2}+\left(9 Z_{s f l}{ }^{2}-\frac{1}{9} Z_{s f l}{ }^{2}\right)^{2}} \\
& Z_{s 3 h} \cong 2.89 Z_{s f l}
\end{aligned}
$$

where $Z_{s 3 h l}$ is the magnitude of the impedance of inductor $L$ for third harmonic components, $Z_{s 3 h c}$ is the magnitude of the impedance of capacitor $C$ for third harmonic components, $Z_{\mathrm{s} 3 \mathrm{~h}}$ is the magnitude of total impedance for the third harmonic component. The (16) is by neglecting $R_{s}$ as it is much less than $Z_{\text {sfl }}$. Similarly, the magnitude of impedances for different harmonics can be calculated. Thus, from (12) and (16) the impedance for the fundamental component is negligible if the proper design is done and the third harmonic has considerably high impedance compared to the fundamental component. Transforming these impedances into the primary side.

$$
Z_{p}=Z_{s}\left(\frac{1}{K}\right)^{2}
$$

Where $Z_{p}$ is impedance transformed into the primary side, $Z s$ is the impedance of LC filter connected on secondary. This impedance $Z_{p}$ will be imposed on each phase. The simplified single-phase equivalent circuit diagram of the THBT is as shown as Figure 3. From the above discussions symmetrical components THBT has effective impedance as the sum of leakage inductance $\mathrm{L}_{\mathrm{k}}$, primary winding resistance $\mathrm{R}_{\mathrm{pw}}$ which is negligibly small. From (12) and (17) the impedance on zero sequence fundamental components imposes extra secondary winding resistance Rs transformed into the primary side, but the third harmonic imposes high impedance $Z_{\mathrm{s} 3 \mathrm{~h}} \cong 2.89 \mathrm{Z}_{\mathrm{sfl}}$ (from (16)) transformed into the primary side. Hence THBT has minimum effect on symmetrical components and zero sequence fundamental components but imposes high impedance on zero sequences higher harmonic especially on dominant third harmonic. So, it is suitable for use in industrial/distribution systems where third harmonic current needs to be blocked without affecting zero sequence currents which are created by single-phase unequal loading.

Figure 4 shows the equivalent magnetic circuit of the THBT. It consists of three MMF (one for each winding) connected in series which are due to zero sequence currents. The MMF due to symmetrical currents cancels each other as the sum of instantaneous currents is zero. $\mathrm{R}_{0}$ is the reluctance of the magnetic core. There is an opposing MMF which is due to a low impedance path for fundamental zero-sequence current. $\mathrm{r}_{\mathrm{lk}}$ is the reluctance due to leakage flux which passes through the air and is not linked with other coils.

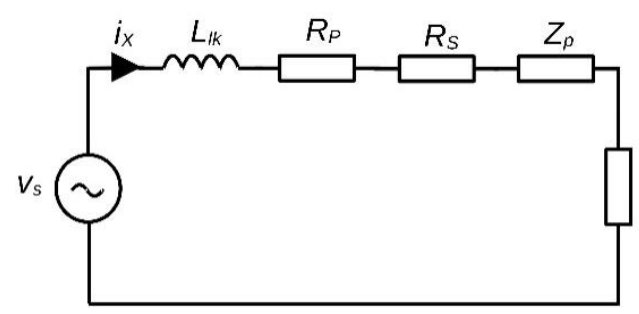

Figure 3. THBT single-phase equivalent circuit

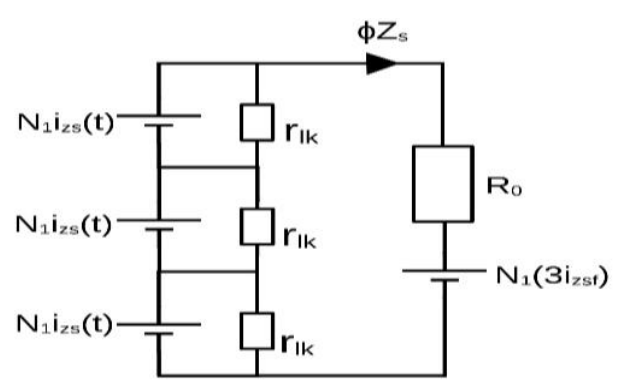

Figure 4. THBT equivalent magnetic circuit 


\subsection{Design considerations}

Some considerations in the research are:

a) The primary conductors should be capable of withstanding load current

b) The secondary conductors should be capable of withstanding zero-sequence current transformed into the secondary side

c) From Figure 4 the THBT should be designed to bear the flux generated due to $3\left(N_{1} i_{z s}(t)-N_{1} i_{z s f}\right)$

d) The inductance of each phase of the THBT is defined by (18),

$$
L_{0}=\frac{N_{1}^{2}}{R_{0}}=\frac{N_{1}^{2} \mu A}{l_{c}}
$$

where N1 is several primary turns, A is a cross-sectional area of the magnetic core, $\mu$ is the permeability of the magnetic core. While designing mutual inductance an important thing to be considered is the saturation of the magnetic core. The maximum flux density should be less than the saturation flux density. For this, the dc flux (due to the DC component in zero sequence) needs to be considered. Also, the flux due to the symmetrical component is zero, and flux due to zero sequence fundamental components is also zero. Hence it can be written as (19).

$$
B_{m}=B_{d c}+B_{a c}
$$

Where,

$$
B_{d c}=3 \frac{I_{z s d c} N_{1}}{A R_{0}}=3 \frac{I_{z s d c} N_{1} \mu A}{A l_{c}}=3 \frac{I_{z s d c} N_{1} \mu}{l_{c}}
$$

we know that $V=4.44 \phi f N$, substituting the components,

$$
V_{z s t h}=4.44 B_{a c} A * 3 f N_{1}
$$

Re-writing (21)

$$
B_{a c}=\frac{V_{z s t h}}{13.32 A f N_{1}}
$$

Substituting (20) and (22) in (19)

$$
B_{m}=3 \frac{I_{z s d c} N_{1} \mu}{I_{c}}+\frac{V_{z s t h}}{13.32 A f N_{1}}
$$

Thus the design of mutual inductance should be designed such that both (18) and (23) are met.

a) The inductor $L$ and capacitor $C$ should be capable of withstanding current $i_{z s}$.

b) The value of filter elements $\mathrm{L}$ and $\mathrm{C}$ are chosen such that they resonate at the fundamental frequency, $\frac{1}{2 \pi \sqrt{L C}}$ Figure 5 shows the relation of magnitude of impedances on the selection of $\mathrm{L}$ and $\mathrm{C}$. If higher the value of the capacitor, lower the value of the inductor and hence lower the impedance at third harmonic. If lower the capacitor value, the higher the inductor and hence higher the impedance at the third harmonic. The current in the secondary can be written as $i_{2}=\frac{i_{z s}}{K}$. The energy stored in the inductor is given by (24).

$$
E_{L}=\frac{L}{2} \frac{i_{z s p}{ }^{2}}{K^{2}}
$$

From (17) the impedance on the primary side on the third harmonic current is decreased by the square of the turn's ratio but by (24) energy stored in the inductor decreases by the square of the turns ratio. That means if the higher the turn's ratio, the lower the energy stored and hence lower the cost of an inductor, as cost and size of inductor depend on maximum energy stored in the inductor. But impedance on the third harmonic also decreases which is not desired. If lower the turn's ratio, energy stored increases, and hence cost also increases. But impedance on third harmonic also more. Hence there should be optimization depending on the amount of fundamental zero-sequence current for which the THBT needs to be designed, the impedance on the third harmonic required and the cost of the system.

c) While designing the value of $\mathrm{L}$ and $\mathrm{C}$ the leakage inductance of the transformer should be considered. Hence adjustable inductance is a better option Figure 5. 


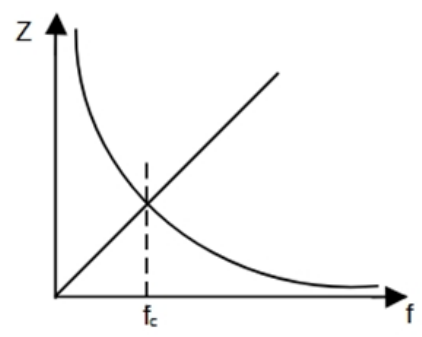

Figure 5. Resonance impedance

\subsection{Implementation using three single-phase transformers}

The THBT can be implemented using three single-phase transformers. Figure 6 is the circuit diagram of THBT using three single-phase transformers. In this type of implementation, the primary transformer is connected in series with the supply, and the secondary is connected in parallel. Across the output of the secondary winding, an LC filter that passes fundamental frequency current is connected.

The THBT can be implemented using three single-phase transformers. Figure 6 is the circuit diagram of THBT using three single-phase transformers. In this type of implementation, the primary transformer is connected in series with the supply, and the secondary is connected in parallel. Across the output of the secondary winding, an LC filter that passes fundamental frequency current is connected. Applying KCL on secondary side,

$$
\begin{aligned}
& i_{a 2}+i_{b 2}+i_{c 2}=i_{\text {filter }} \\
& \frac{N_{2}}{N_{1}}\left(i_{a}+i_{b}+i_{c}\right)=i_{\text {filter }} \\
& \frac{N_{2}}{N_{1}}\left(3 i_{z s}\right)=i_{\text {filter }}
\end{aligned}
$$

Where $\mathrm{i}_{\mathrm{a} 2}, \mathrm{i}_{\mathrm{b} 2}$, and $\mathrm{i}_{\mathrm{c} 2}$ are secondary currents, $\mathrm{i}_{\mathrm{a}}, \mathrm{i}_{\mathrm{b}}$, and $\mathrm{i}_{\mathrm{c}}$ are primary currents. As the sum of instantaneous current of symmetrical currents is zero, the voltage across filter corresponding to symmetrical components is also zero and hence the impedance on symmetrical components is zero. The impedance on zero sequences is similar to (13) to (17). The equivalent circuit by transforming into the primary side is similar to Figure 3, except the secondary winding resistance is not equal to zero for symmetrical components as there is the flow of symmetrical components $\frac{N_{2}}{N_{1}} i_{a}, \frac{N_{2}}{N_{1}} i_{b}, \frac{N_{2}}{N_{1}} i_{c}$ on the secondary side.

Hence while designing transformers the secondary side conductor size should be selected such that it should be able to withstand symmetrical components also. The equivalent magnetic circuit of each singlephase transformer is as shown in Figure 7, where $\mathrm{N}_{1} \mathrm{i}_{\mathrm{x}}$ is the MMF due to primary side current $\left(\mathrm{i}_{\mathrm{a}}, \mathrm{i}_{\mathrm{b}}\right.$, and $\mathrm{i}_{\mathrm{c}}$ ). $\mathrm{N}_{1}\left(\mathrm{i}_{\mathrm{x} \text { symmetrical }}+\mathrm{i}_{\text {sffundamental }}\right)$ is opposing MMF which are produced due to current on the secondary side due to low impedance. $\mathrm{R}$ is the reluctance of the magnetic path and $\mathrm{r}_{\mathrm{k}_{\mathrm{k}}}$ - the reluctance of leakage inductance which is due to flux linkage through the air.

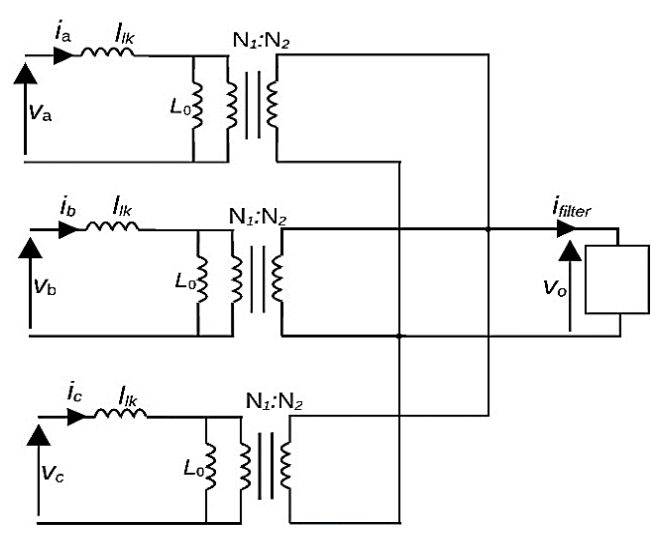

Figure 6. THBT uses three single-phase transformers

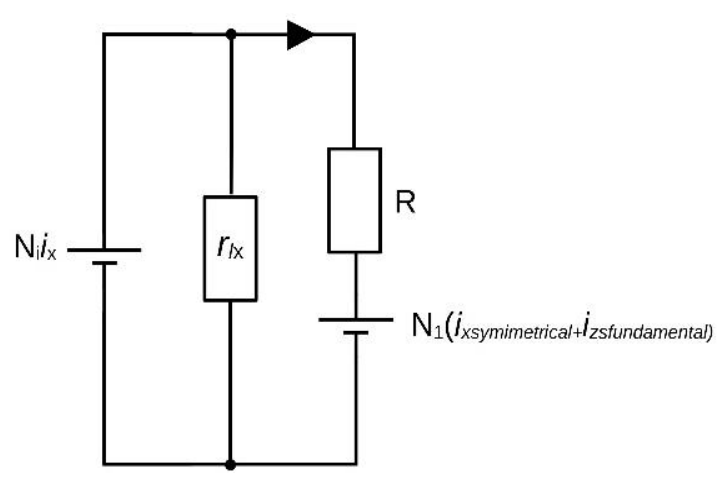

Figure 7. Magnetic equivalent circuit of each singlephase transformer 


\section{RESULTS AND DISCUSSION}

There are two types of scenarios present. First, the supply contains zero-sequence voltages, and third harmonic voltages. The second, the load is asymmetric and unbalanced which consumes zero sequence current, and the third harmonic currents. For our study purpose, four simulations are performed under three conditions: with THBT, with ZSBT, and without any filter. To build the THBT, three single-phase transformers have been used. These transformers have a leakage inductance $\mathrm{L}_{\mathrm{lk}}$ of $1 \mathrm{mH}$, winding resistance of both the windings as $r_{\text {zsbt }}$ of $0.1 \Omega$, and a magnetizing inductance L0 of $2.9 \mathrm{H}$. The filter inductor $\mathrm{L}$ of $1 \mathrm{H}$ and capacitor of $10.13211 \mu \mathrm{F}$ are taken. The ZSBT is formed similar to THBT but without a fourth winding and L-C filter. The simulation results are plotted and observed only for steady-state conditions from 1 second to 1.2 seconds.

Case1:

In this case, the star-connected 3phase square wave supply (RMS voltage of $220 \mathrm{~V}, 50 \mathrm{~Hz}$ ) is taken. The load is a balanced resistive load with $\mathrm{R}=100 \Omega$. Simulations are carried out with THBT, with ZSBT, and without any filter. The simulation reports are as shown in Figure 8 and Figure 9. Figure 10 shows harmonic analysis of load voltages.

From Figure 8, the voltage across THBT fourth winding contains only third harmonic voltages. It is observed that the THBT imposes high impedance on the $3^{\text {rd }}$ harmonic and hence the voltage across the THBT filter is majorly $3^{\text {rd }}$ harmonic. Figure 9 shows the load current and voltages with THBT and with ZSBTcontain very less $3^{\text {rd }}$ harmonics. The load voltage without a filter is the same as the power supply and load current follows the voltage as the load is resistive. From Figure 10 it is observed that the zero-sequence harmonic voltages like $3^{\text {rd }}, 9^{\text {th }}$, and $15^{\text {th }}$ are filtered with THBT and with ZSBT filter. Both THBT and ZSBT are found good in eliminating zero-sequence harmonic under this case.

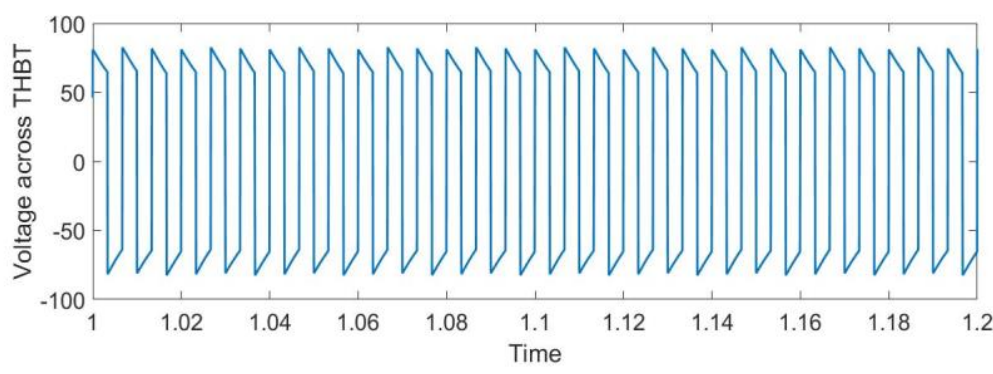

Figure 8. The voltage across THBT fourth winding or L-C filter

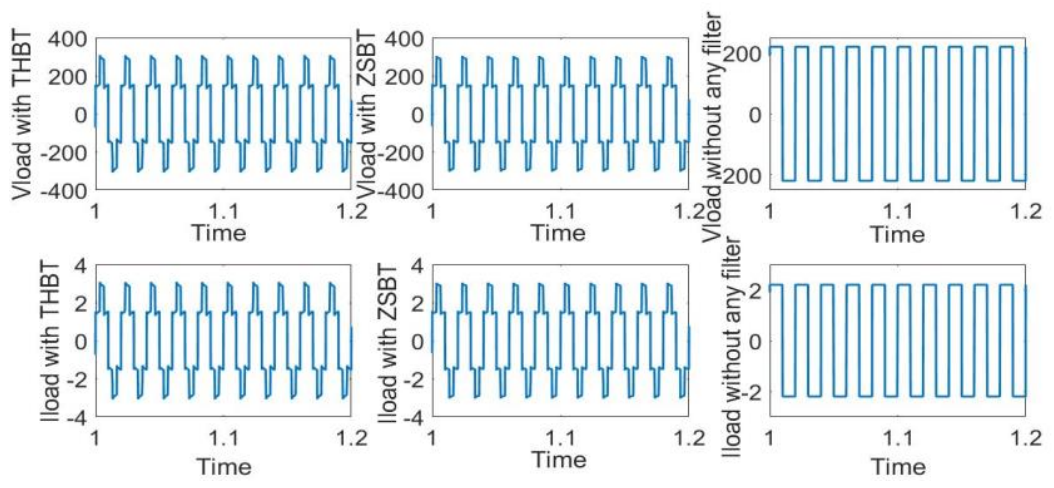

Figure 9. Load voltage and current waveforms with THBT (left), with ZSBT (middle), and without any filter (right)

\section{Case2:}

In this case, the supply voltage is 3-phase voltage (RMS 220V) with no harmonic voltages but with $20 \%$ of fundamental zero-sequence voltage is taken. The load is a balanced resistive load with $\mathrm{R}=100 \Omega$. The simulation is carried out with THBT, with ZSBT, and without any filter. 
From Figure 11 it can be observed that the voltage across the THBT LC filter is very less. LC filter offers zero impedance at resonating frequency. But a small voltage appears across the THBT LC filter due to winding resistance. The load voltages with THBT and without filter are the same as supply as shown in Figure 12 which contains a $20 \%$ zero sequence component. The load voltages with ZSBT contain very few zero-sequence components. Similarly from Figure 13, load currents with THBT and without filter are the same which contain $20 \%$ zero-sequence components. The load current with ZSBT contains very few zerosequence components. Thus, it is evident that THBT does not affect the fundamental component of supply even if the supply contains zero-sequence components whereas ZSBT filters zero-sequence components even fundamental also. In distribution systems, if zero-sequence components are present in the power supply, THBT will not produce any effect on the power supply voltage at the load. But, ZSBT will alter the supply voltage which is not desired. Thus, THBT is found better than ZSBT in this case.

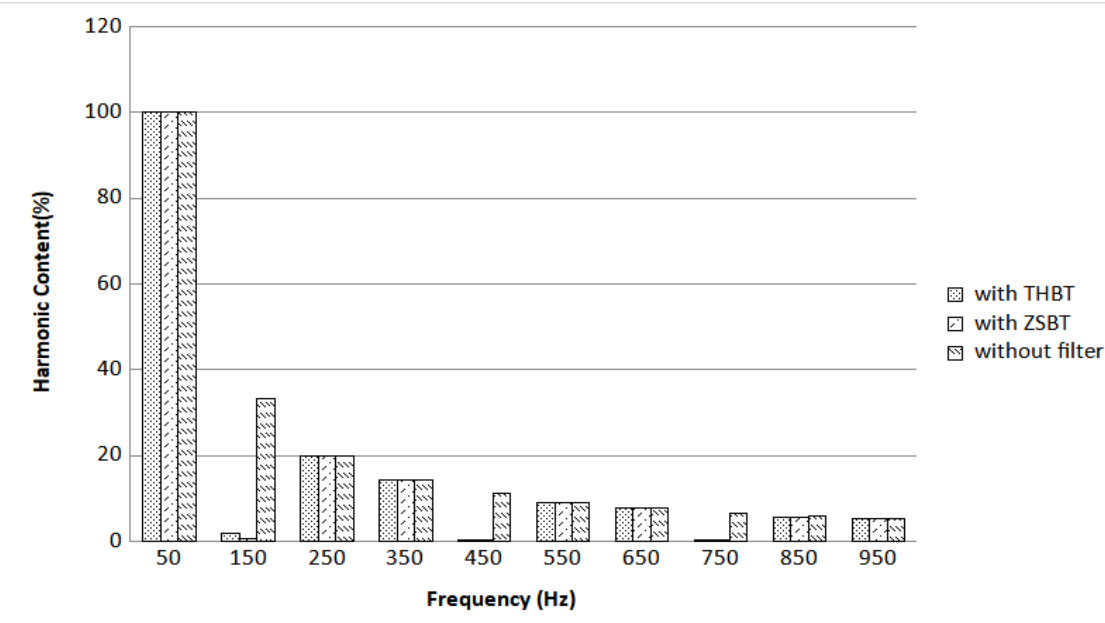

Figure 10. FFT analysis of load voltage with THBT, with ZSBT, and without any filter

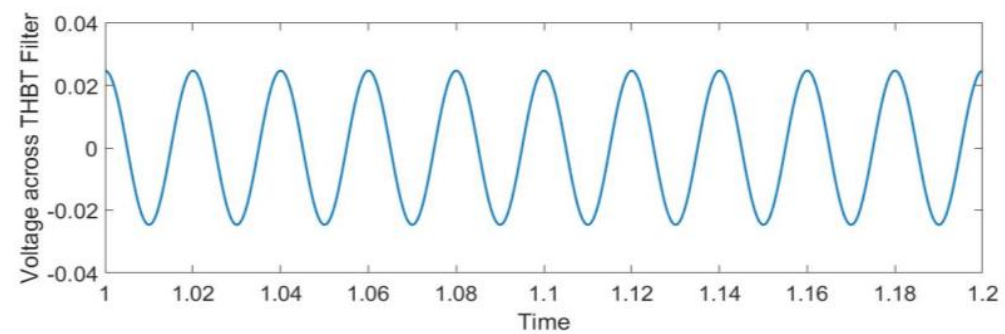

Figure 11. Voltage across THBT
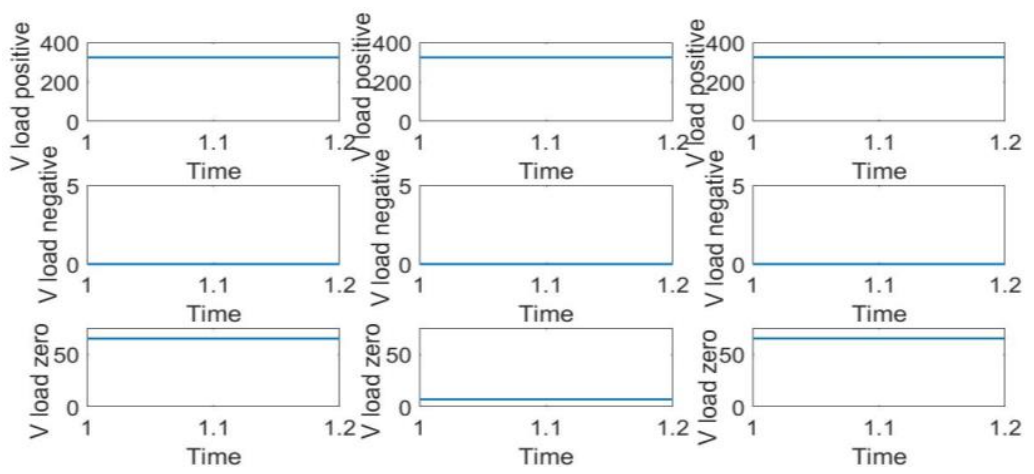

Figure 12. Symmetrical analysis of load voltage with THBT (left), with ZSBT (middle), and without any filter (right) showing positive, negative, and zero sequence peak voltages 

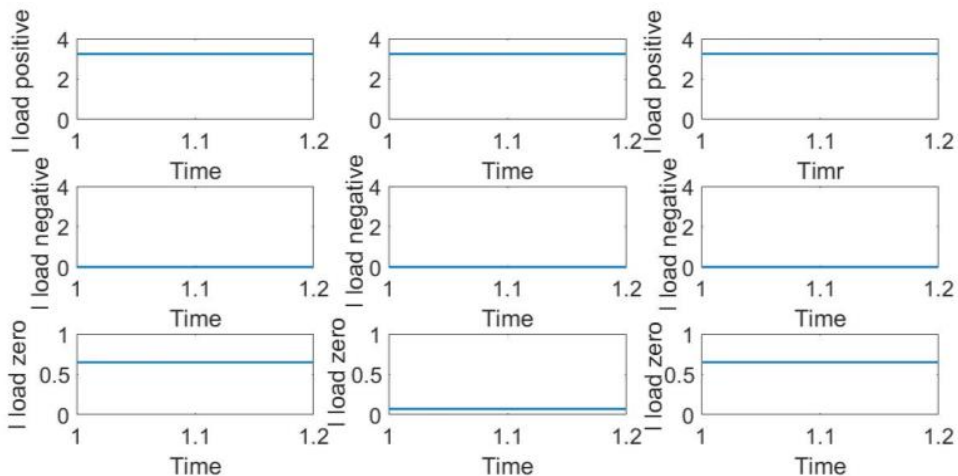

Figure 13. Symmetrical analysis of load current with THBT (left), with THBT(middle), and without any filter (right) showing positive, negative, and zero sequence peak currents

\section{Case3:}

In this case, the supply voltage is 3-phase voltage (RMS 220V) with no harmonic component. The load is balanced resistive load with $\mathrm{R}=100 \Omega$ and for phase $\mathrm{B}$, a bridge rectifier with filter capacitor of $1000 \mu \mathrm{F}$ and load resistance of $1000 \Omega$ is connected. The simulation is carried out with THBT, with ZSBT, and without any filter. From Figure 14, the load current without a filter has a high surge current up to $20 \mathrm{~A}$ at the peak due to the forward conduction of rectifier diodes. The amplitude of surge current reduced drastically to under 5A with THBT and with ZSBT.

Figure 15 shows the effect of the rectifier load on phase B voltage with a clipping at peak. The drop in the peak voltage at phase $\mathrm{B}$ is due to the high rate of change of current during the conduction of rectifier diodes. This voltage drop in phase B is induced in the other two phases because of the mutual coupling of windings. Hence peak load voltages at the other two phases are also varied. With ZSBT the load voltages are similar to THBT waveforms. Thus, both THBT and ZSBT are found equally effective in this case.

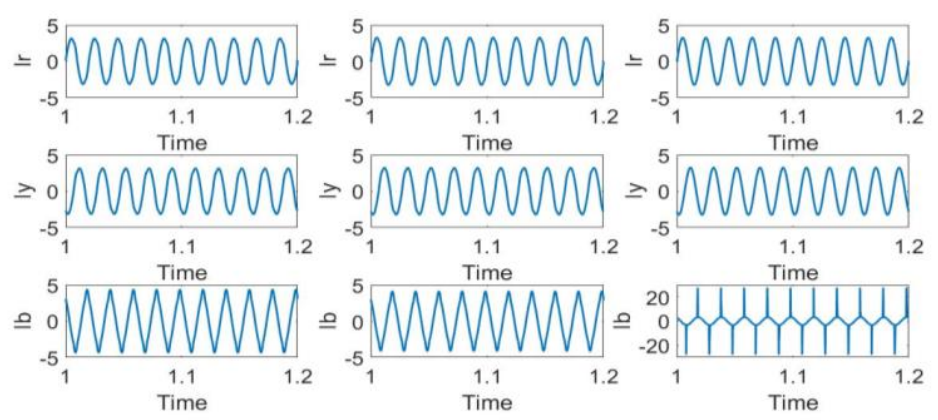

Figure 14. Load current with THBT (left), with ZSBT (middle), and without any filter (right)

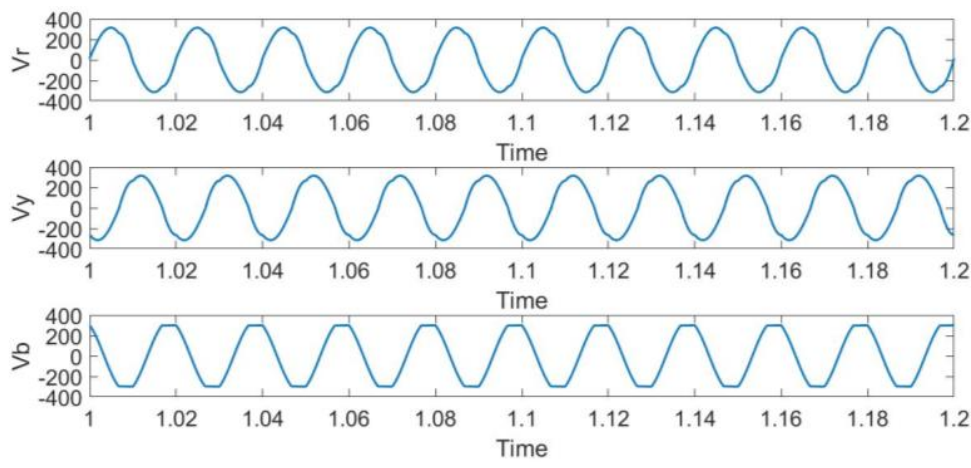

Figure 15. Load voltage with THBT 


\section{Case4:}

In this case, the supply voltage is 3-phase voltage (RMS 220V) with no harmonic. The load is balanced resistive load with $\mathrm{R}=100 \Omega$ and three AC sources of $1 \mathrm{~A}$ each which are in phase are connected in parallel with load resistance $\mathrm{R}$ on each phase. This constitutes zero-sequence loads for simulation purposes.

Figure 16 shows the voltage across the THBT LC filter. Since the LC filter resonates at the fundamental frequency, it offers zero impedance. But due to winding resistance, a small voltage appears across the THBT LC filter. From Figure 17, the load currents with THBT and without filter are similar. With ZSBT the load current has less zero-sequence component since it blocks zero-sequence. This is a nondesirable operation from ZSBT under this case. In Figure 18, it is observed that with THBT, the supply voltage appears on the load side. There is only a positive-sequence component in load voltages. With ZSBT, the load voltage has a zero-sequence component that is induced due to zero-sequence current components of the unbalanced load. This undesired zero-sequence voltage component induced due to ZSBT makes the load voltage unbalanced. Hence THBT is found more effective in this case.

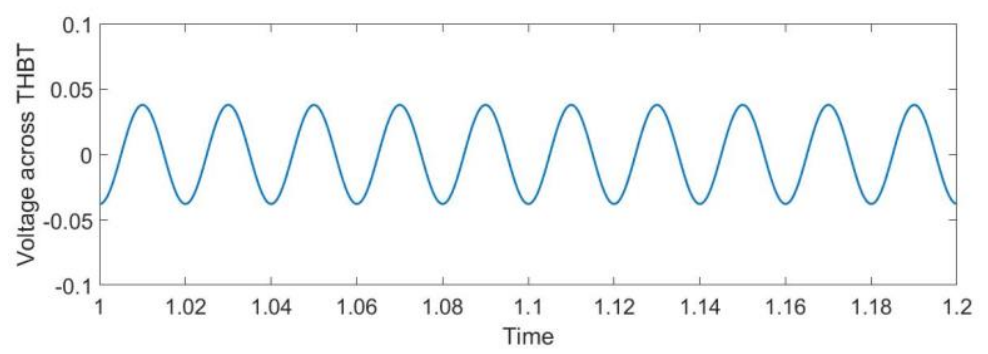

Figure 16. The voltage across the THBT LC filter
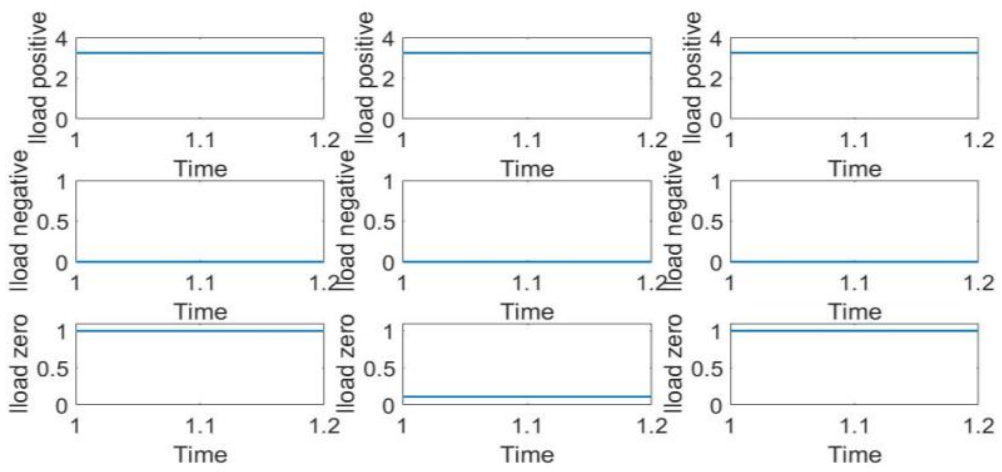

Figure 17. Symmetrical analysis of load currents with THBT with ZSBT and without any filter
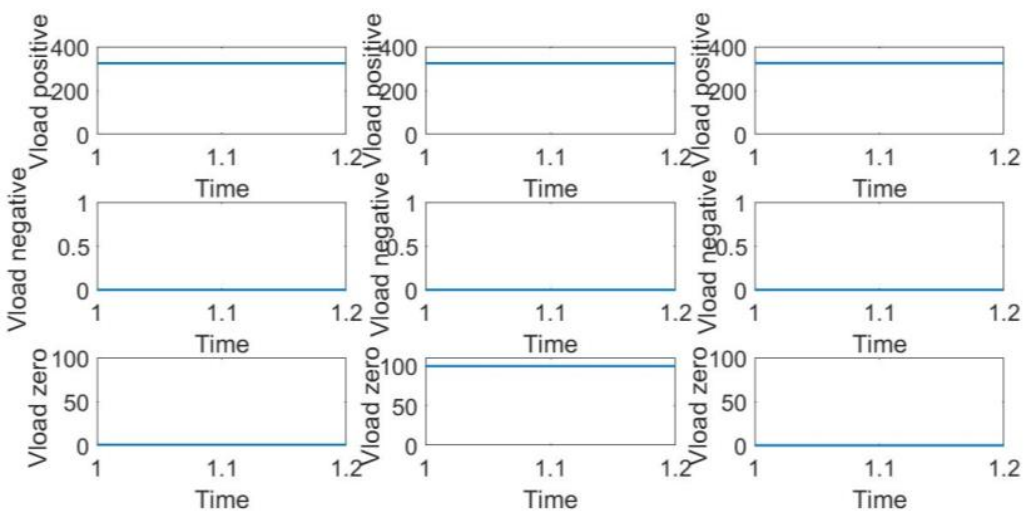

Figure 18. Symmetrical analysis of load voltage with THBT (left), with ZSBT (middle), and without any filter (right) 


\section{CONCLUSION}

From case 1 simulations, it is observed that THBT can be used in 3 phase systems where the supply has 3rd harmonic voltage. Both THBT and ZSBT are found good in eliminating zero-sequence harmonic under this case. From case 2 simulations, it is clear that the THBT imposes negligible impedance for 3-phase systems where the supply has fundamental zero-sequence voltage. The same supply voltage appears across the load without any drop due to fundamental zero-sequence voltage. If zero-sequence components are present in the power supply, THBT will not produce any effect on the power supply voltage at the load. But, ZSBT will alter the supply voltage which is not desired. Thus, THBT is found better than ZSBT in this case. From case 3 simulations, it can be observed that, if a non-linear load like bridge rectifier is present at the load, the THBT suppresses the peak current drastically. But the supply voltage gets distorted at all the phases. Since THBT is a coupled inductor, the voltage induced due to load current is coupled with all the windings. Simulations show that both THBT and ZSBT are found equally effective in this case. From case4 simulations, it can be understood that the THBT does not affect load voltage due to zero sequence components of load current which usually happens due to single-phase loading in distribution systems. With ZSBT, the load voltage has a zero-sequence component making the load voltage unbalanced, and also ZSBT alters load current. Hence THBT is found more effective in this case. Thus THBT is a better option to minimize third harmonic current in 3-phase systems and to minimize the copper loss in the neutral line.

\section{ACKNOWLEDGEMENTS}

The present work has been filed for patent application with the Indian Patent Office Application No. “202141020237”, Filing Date: May 3, 2021.

\section{REFERENCES}

[1] A. Laka, J. A. Barrena, M. A. R. Vidal, and G. Calvo, "Novel Zero-Sequence Blocking Transformer (ZSBT) Using Three SinglePhase Transformers," IEEE Transactions On Energy Conversion, vol. 28, no. 1, pp. 234-242, 2013, doi: 10.1109/TEC.2012.2229282.

[2] A. Laka, J. A. Barrena, J. C. Zabalza, and G. Calvo, "IP-ZSBT Magnetic Configuration for Parallelization-Serialization of ThreePhase High Power Converters," IEEE Transactions On Energy Conversion, vol. 29, no. 2, pp. 366-374, 2014, doi: 10.1109/TEC.2014.2304395.

[3] D. A. Paice, "Transformers For Multipulse AC/DC Converters," United States Patent 6,101,113, 8 Aug 2000.

[4] L. Basabe and Aitor, "Transformer for blocking zero sequence components," Europe Patent EP 2579280 A1, 10 April 2013.

[5] C. M. Hadzer, S. Masri, and N. Mahamad, "A Study on Zero-passing Transformer in Harmonics Reduction," in National Power and Energy Conference (PECon), Bangi, Malaysia, 2003, doi: 10.1109/PECON.2003.1437440.

[6] Q. Song, Z. Yin, J. Xue, and L. Zhou, "Zero-Sequence Harmonics Current Minimization Using Zero-Blocking Reactor and ZigZag Transformer," in DRPT, Nanjing, China, 2008, doi: 10.1109/DRPT.2008.4523691.

[7] V. B. Bhavaraju and P. N. Enjeti, "An Active Line Conditioner to Balance Voltages in a Three-phase System," IEEE Transactions on industrial applications, vol. 32, no. 2, pp. 287-292, 1996, doi: 10.1109/28.491476.

[8] M. Ahmadi, H. Samet, and T. Ghanbari, "Discrimination of internal fault from magnetising inrush current in power transformers based on sine-wave least-squares curve fitting method," IET Science, Measurement \& Technology, vol. 9, no. 1, pp. 73-84, 2015, doi: 10.1049/iet-smt.2014.0012.

[9] A. M. Kuparev, I. I. Litvinov, and D. V. Baklanov, "Harmonic Analysis of the Currents in the Power Transformer Differential Protection Circuits in the Cases of External and Internal Faults," In 2018 XIV International Scientific-Technical Conference on Actual Problems of Electronics Instrument Engineering (APEIE), 2018, pp. 202-209, doi: 10.1109/APEIE.2018.8545971.

[10] M. E. Gamal, H. Dessouki, and A. Lotfy, "Implementation of Transformer Static Differential Relay with Harmonic Blocking. (Dept. E)," MEJ. Mansoura Engineering Journal, vol. 31, no. 1, pp. 62-66, 2020, doi: 10.21608/BFEMU.2020.129250.

[11] R. P. Medeiros and F. B. Costa, "A wavelet-based transformer differential protection: internal fault detection during inrush conditions." IEEE Transactions on Power Delivery, vol. 33, no. 6, pp. 2965-2977, 2018, doi: 10.1109/TPWRD.2018.2852485.

[12] K. Behrendt, N. Fischer, and C. Labuschagne, "Considerations for using harmonic blocking and harmonic restraint techniques on transformer differential relays," In proceedings of the 33rd Annual Western Protective Relay Conference, 2006.

[13] J. O. Aibangbee and S. O. Onohaebi, "Power Transformer Differential Relay Inrush Restraint Setting Applications," IOSR Journal of Electrical and Electronics Engineering (IOSR-JEEE), vol. 11, no. 1, pp. 68-75, 2016, doi: 10.9790/1676-1101046875.

[14] B. Jiří, and P. Martin, "Transformer inrush-Harmonics in the current." In 2018 19th International Scientific Conference on Electric Power Engineering (EPE), 2018, pp. 1-4, doi: 10.1109/EPE.2018.8396014.

[15] W. Jiang, W. Ma, J. Wang, W. Wang, X. Zhang, and L. Wang. "Suppression of zero sequence circulating current for parallel three-phase grid-connected converters using hybrid modulation strategy," IEEE Transactions on Industrial Electronics, vol. 65, no. 4, pp. 3017-3026, 2017, doi: 10.1109/TIE.2017.2750625.

[16] S. K. Shaikh, A. M. Mulla, S. U. Bagwan, and Y. A. Makandar, "Analysis and Application of Zigzag Transformer in Distribution System for Mitigation of Triplen Harmonics," In 2020 th International Conference on Communication and Electronics Systems (ICCES), 2020, pp. 94-98, doi: 10.1109/ICCES48766.2020.9137857.

[17] A. Rahman, M. M. Rahman, and M. R. Islam, "A study of THD minimization of three phase inverter using modified THPWM," In 2017 3rd International Conference on Electrical Information and Communication Technology (EICT), IEEE, pp. 1-4, 2017, doi: 10.1109/EICT.2017.8275225.

[18] A. M. Eltamaly, "A novel current injection device for harmonic reduction of three-phase controlled converters in renewable energy utility interfacing," Journal of Renewable and Sustainable Energy, vol. 9, no. 4, p. 045504, 2017, doi: 10.1063/1.4997494. 
[19] M. S. Almutairi and S. Hadjiloucas, "Harmonics Mitigation Based on the Minimization of Non-Linearity Current in a Power System." Designs, vol. 3, no. 2, p. 29, 2019, doi: 10.3390/designs3020029.

[20] S. L. Gbadamosi, N. I. Nwulu, and Y. Sun, "Harmonic and power loss minimization in power systems incorporating renewable energy sources and locational marginal pricing," Journal of Renewable and Sustainable Energy, vol. 10, no. 5, p. 055501, 2018, doi: $10.1063 / 1.5041923$.

[21] A. K. M. Arafat and S. Choi, "Active current harmonic suppression for torque ripple minimization at open-phase faults in a fivephase PMa-SynRM," IEEE Transactions on Industrial Electronics, vol. 66, no. 2, pp. 922-931, 2018, doi: 10.1109/TIE.2018.2829685.

[22] A. Routray, R. K. Singh, and R. Mahanty, "Harmonic minimization in three-phase hybrid cascaded multilevel inverter using modified particle swarm optimization," IEEE Transactions on Industrial Informatics, vol. 15, no. 8, pp. 4407-4417, 2018, doi: 10.1109/TII.2018.2883050.

[23] A. Yadav, S. N. Singh, and S. P. Das, "Analysis of transformerless MMC and suppression of third order harmonic current," In 2019 National Power Electronics Conference (NPEC), IEEE, 2019, pp. 1-6, doi: 10.1109/NPEC47332.2019.9034733.

[24] A. M. Abd-Elrasool Elagab and I. M. El-Amin, "Minimization of Harmonics Penetration into Transmission and Distribution Systems by Utilizing Tertiary Winding of the Transformer," In 2017 9th IEEE-GCC Conference and Exhibition (GCCCE), IEEE, 2017, pp. 1-9, doi: 10.1109/IEEEGCC.2017.8448241.

[25] S. Krishnamurthy and B. E. Baningobera, "IEC61850 standard-based harmonic blocking scheme for power transformers," Protection and Control of Modern Power Systems, vol. 4, no. 1 p. 10, 2019, doi: 10.1186/s41601-019-0123-7.

\section{BIOGRAPHIES OF AUTHORS}
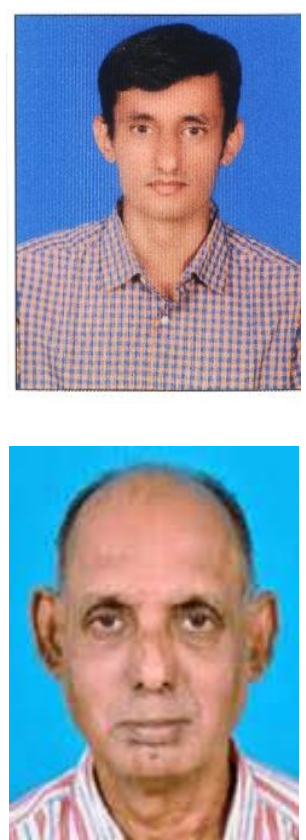

Mr. Vishnuprasada Vittal Bhat (D) 81 SC $P$ received B.E. Degree in Electrical and Electronics in 2006 from NMAMIT, Nitte. He started a manufacturing unit"Swadeshee" of electronic gadgets in 2007 and did is M-Tech in Power Elctronics in St. Joseph Engineering College, Vamanjoor in 2014. He continued his business and founded agtech strtup company "Krishi-Tantra" (Klonec Automation Systems Pvt Ltd) in 2017. His area of interest is Power electronics, Power systems and Embedded Systems. He has applied for three patents of his innovations "A Soil Analysis Apparatus" (Application number: 202047035847 A), "An Electrical Transformer For Suppressing Triplen Harmonic Distortion In Input Electrical Power And Method Thereof" along with team mates (Application number: 202141020237) and "An Automated Soil Analysis Apparatus" (Application number: 202141022299) He is engaged as a research scholar in NMAMIT Nitte. He can be contacted at email: vishnupangala@gmail.com.

Dr. Pinto Pius (iD 8 SC P is the former professor in the department of Electrical and Electronics Engineering NMAMIT, Nitte. His teaching and research focus is on power electronics and control systems. He received his B.E. in Electrical Engineering from Mysore University (1976), M-Tech from Mangalore University (1999) and Ph.D. in Power Electronics from NITK Surathkal (2008). He has an industrial experience of 26 years and teaching experience of 13 years. He can be contacted at email: loypinto@yahoo.com, alternative id is email: pintopaj@nitte.edu.in. 